\title{
Development of a Biosensor for the Prediction and Early Detection of Cardiovascular Diseases Based on Saliva Composition Analysis
}

\author{
Victor Aleksandrovich Stupin ${ }^{1}$, Ekaterina Vladimirovna Silina ${ }^{2}$, \\ Dmitry Valerievich Kolesov ${ }^{3}$, Igor Vladimirovich Yaminsky \\ and Valery Semenovich Shalygin
}

\author{
${ }^{1}$ Pirogov's Russian National Research Medical University , \\ Ostrovityanova str, 1, Moscow, 117997, Russia \\ ${ }^{2}$ I.M. Sechenov First Moscow State Medical University, Trubetskaya str, 8, Moscow, 119991, Russia \\ Ohe Institute of General Pathology and Pathophysiology, Baltiyskaya st., 8, Moscow, 125315, Russia \\ ${ }^{4}$ M.V. Lomonosov Moscow State University, Leninskiye Gory, 1, GSP-1, Moscow, 119991, Russia \\ ${ }^{5}$ Moscow Aviation Institute (National Research University) Volokolamsk
} Highway, d. 4, Moscow, A-80, GSP-3, 125993

DOI: http://dx.doi.org/10.13005/bbra/1857

(Received: 28 September 2015; accepted: 01 November 2015)

\begin{abstract}
The aim of the study was the development of a biosensor for prediction and early detection of diseases, including myocardial infarction, based on the analysis of human saliva composition with implanted biosensor devices. For the implementation of the stated objective a device enabling real-time detection of small concentrations of proteins in specific liquid mediums was developed. An evaluation of biosensor sensitivity was performed experimentally with cardiac marker - C-reactive protein, which demonstrates an increase of concentration in all non-specific inflammatory reactions, including myocardial infarction and other acute cardiovascular accidents. A receptor layer was developed based on monoclonal antibodies, enabling specific binding of $\mathrm{C}$-reactive protein, generating lateral strain in the layer. The feasibility of $\mathrm{C}$-reactive protein detection in fluid flow using microcantilever sensors was demonstrated. To allow the possibility of sensor device implantation into oral cavity for continuous monitoring and early detection of diseases based on human saliva composition, a concept and prototype of microcapsule transducer was developed. The transducer constitutes a microaspirator pump with the feature of operator chamber rinsing, which provides circulation of biological fluid samples in the implanted device.
\end{abstract}

Key words: cantilever sensor, saliva, cardiac markers, micropump,

C-reactive protein, constant monitoring.

Increasing costs of healthcare and overall population ageing determine the growing need for the development of technologies and devices for remote continuous monitoring of health parameters of population. Utilization of implantable and nonimplanted biosensors potentially may improve medical care provision for patients with remote

\footnotetext{
* To whom all correspondence should be addressed.
}

real-time monitoring of certain health parameters. In the last decades a lot of applied research was performed, leading to the development of biosensors, detecting the changes of certain markers in biological fluids for screening and diagnostics.

In view of these factors a proper multidisciplinary work was carried out, aimed at the development of a biological sensor for the prediction and early detection of cardiovascular diseases based on the analysis of human saliva 
composition. It is assumed that a biosensor implanted into the oral cavity, designed to perform dynamic analysis of low concentrations of proteins in the saliva, will provide patient health status monitoring and in case of critical changes of cardiac markers will immediately alert the patient and the attending physician about the impending danger, sending the information into a healthcare facility before irreversible changes in the heart tissue take place. This may help to prevent cardiovascular mortality and disability, improve the results of treatment using dynamic monitoring, and increase the efficacy of pharmacological therapy with appropriate correction and timely therapeutic interventions, which can translate into increased life expectancy and improved quality of life.

The developed device is based on a microcantilever sensor. Previous studies demonstrated that the most valuable predictors of an approaching cardiovascular accident are cardiac markers - cardiac troponin I, creatine kinase-MB, myoglobin C-reactive protein, soluble intracellular adhesion molecular protein-1, interleukin-1-â, fatty acid binding protein [Silina E.V. \& Stupin V.A., 2014]. That is why on that stage of work we experimentally demonstrated the feasibility of detection of different concentrations of C-reactive protein (CRP) in a fluid flow using microcantilever sensor method. CRP was chosen as a universal prognostic marker of non-specific inflammatory reactions including myocardial infarction. A microcapsule transducer was developed to provide the ability of implantation into the oral cavity.

\section{Microcantilever sensors}

Microcantilevers are thin and flexible silica plates (consoles), anchored with one side on a massive base. Thanks to its ultrathin structure (approx. 1 micrometer) these microcantilevers are highly sensitive to external force impact and easily bend under the influence of extremely weak forces, comparable to intermolecular forces. Current technology enables the measurement of such a curve with an accuracy of $1 \mathrm{~nm}$ or higher.

Early studies of microcantilever use for chemical detection were performed 20 years ago in IBM research laboratory (Zurich, Switzerland) and Basel University [Gimzewski J.K. et al., 1994]. Authors demonstrated static curve of cantilever as a result of catalytic reaction of hydrogen and oxygen into water with high sensitivity. The first utilization of cantilever sensors for biological systems was demonstrated in 1996, in non-specific bovine serum albumin adsorption [Butt H.J., 1996]. Since that time different researchers demonstrated the potential of micromechanic cantilevers for the development of highly sensitive chemical and biological sensors [Boisen A. and Thundat T., 2009; Fritz J. et al., 2000; Fritz J., 2008]. Thus, using cantilevers the ability to detect singular mismatch of base pairs between the DNA-probe and sensory DNA was demonstrated [Fritz J. et al., 2000]. A dramatic result of cantilever sensor utilization for the detection of nucleic acids was the detection of non-amplified RNA in the total RNA mass of the cell with threshold of detectability at $10 \mathrm{pM}$ [Zhang J. et al., 2006]. Besides high sensitivity, this experiment demonstrated high selectivity of the method, as it was performed under the conditions of high background noise of non-specific molecules.

Antibody interaction is traditionally used for protein detection with the utilization of cantilever sensors. Detection of two forms of prostate-specific antigen (PSA), which is a marker of prostate cancer, in a clinically significant range of $0.2 \mathrm{ng}-60 \mu \mathrm{g} / \mathrm{l}$ was demonstrated in the presence of human serum albumin [Wu G. et al., 2001]. Rabbit antibodies to human PSA, covalently bound to a golden surface of cantilever using thiol chemistry were utilized as a receptor layer. Abutment cantilever was not used in the study; thermostatic control using Peltier thermoelectric module was performed to eliminate temperature effects.

A series of studies demonstrated detection of proteins, which are markers of cardiovascular diseases. Thus, a simultaneous continuous detection of two cardiac markers ñreatine-kinase and myoglobin was demonstrated [Arntz Y. et al., 2001]. Myoglobin sensitivity was $20 \mu \mathrm{g} / \mathrm{ml}$. Researchers utilized an abutment cantilever to eliminate the effect of non-specific fluctuations with calculation of relative deflection of sensory cantilever. Authors used a continuous flow of the medium, which led to decrease of protein detection time.

Studies describe a sensor based on a microcantilever with laser-optic registration system for the detection of CRP. In the experimental study a triangle cantilever $200 \mu \mathrm{m}$ was used, etched out on a substrate material and placed in a chamber 
sized $70 ̃ 7 \mathrm{~mm}$ from polydimethylsiloxane (PDMS), that is the sensor was made very compact. The limiting factor for the development of a miniature device was the laser-optic system of deflection detection. The range of concentrations detected was $1-500 \mu \mathrm{g} / \mathrm{ml}$ [Chen C.H. et al., 2009]. Distinctive feature of the device is the capability of wireless transmission of analytic signal. Authors also used a low-frequency alternating voltage with $1 \mathrm{~V}$ amplitude for the destruction of antigen-antibody complexes. This enabled sensor regeneration for further utilization. This type of regeneration method demonstrated high efficacy compared to traditional chemical methods, which damage the sensory layer, decreasing sensor sensitivity. In 2013 another sensor for CRP detection was introduced, equipped with an electric system of deflection registration, based on a piezoelectric sensor. This enabled a significant decrease of device dimensions and its complete assembly on a printed board. The measured protein concentration range was 1-200 $\mu \mathrm{g} / \mathrm{ml}$ [Yen Y.K. et al., 2013].

Apart from micromechanic sensors during the recent year were presented new sensors for CRP detection, based on a surface plasmon resonance [Chammem H. et al., 2014]. This method provides a high protein detection limit, at the level of hundreds of nanogram per milliliter, but demands the presence of laser emission source, which may hinder the use of the device in implantable devices. By contrast, cantilever sensors have a promising potential for use in compact autonomous sensors, through the possibility of utilization of piezoelectric and piezoresistive sensors.

The utilization of microcantilever sensors for the detection of biological objects is poorly represented in Russia. However, in the Russian Federation there are research groups, developing other types of micromechanic biosensors [Ivanov M.A., al., 2014; Shashkanova O.Y. et al., 2013; Agafonova L.E. et al., 2012]. Such biosensors include gravimetric piezoquartz immunosensor, quartz microscales. Operating principle of such sensors is based on the change of sensor resonance frequency after attachment of additional mass due to specific binding of analyte from the solution with receptor layer on sensor surface. As sensor signal depends on the mass attached, authors suggested adding nanoparticles of gold to increase method sensitivity. The disadvantage of the method is the need for additional process stages and chemicals, which complicates its utilization in autonomic devices. Besides, gravimetric method is less protected from nonspecific binding, as sensor reacts on any mass attached. Microcantilever sensors we used in our work also have a promising potential for the detection of biological objects by the change of resonance frequency. Microcantilever is a high-Q mechanic resonator. In contrast to gravimetric sensors microcantilevers can detect not only mass changes, but also the change of cantilever stiffness when a solid layer of analyte attaches to its surface. This process can lead to resonant frequency changes, which are significantly greater than those produced by additional mass attachment.

Therefore, cantilever biosensors are promising devices, possessing several unique advantages compared to traditional analytic methods. The do not need the utilization of additional chemicals or tracing, being a direct analytic method. This gives an opportunity of organization of constant monitoring of biological processes. Utilization of chips, containing several consoles with modified different receptor layers permit the performance of a multiple factor analysis with a simultaneous detection of several protein markers, specific for different diseases, including cardiovascular accidents. This undoubtedly will reduce the number of false-positive and falsenegative results during the system operation.

\section{Microcapsule transducers (micropumps).}

An important part of the implantable sensor device is a micropump, producing the flow of analyzed biological fluid through the biosensor.

Micropump technologies are extensively developed and introduced into modern clinical practice. These devices are mainly used for the administration of pharmaceutical products with controlled speed according to a programmed regimen, which allows the attainment of controlled plasma profile of the drug. [Hoshino M. et al., 2009]. An implantable pump usually consists of a container with the pharmaceutical product, micropump, valves, microsensors, microchannels, catheter and appropriate control circuits [Nisar A., 2008; Ashraf M., 2011; Sabashnikov A., 2014]. The first commercially available medical pumps, which are still widely used today, were insulin pumps for the treatment of diabetes. Another area of 
micropump application is intraocular drug administration, when the pump is placed directly on the eye with a small cannula inserted into the anterior or posterior eye chamber through a small incision [Li P.-Y. et al., 2008; Lo R., et al., 2009].

Micropumps for medical diagnostics at present are not widely used. However, considering the growing interest of development engineers and economical and biological utility, it is highly probable that micropumps for diagnostic purposes will appear on the market in the nearest future.

Micropumps can be divided into two main groups - mechanical and non-mechanical devices. A review of worldwide literature on micropumps developed for biologic and medical use [Ashraf M., 2011], demonstrated that the most promising and actively investigated area is the development of mechanical piezoelectric pumps.

\section{METHODS}

The development of the device was performed after an extensive elaboration of patent data and global scientific literature with an analysis of technical solutions. Patent search was performed using different databases (including databases of European, Eurasian, Japanese patent offices, database of the World Intellectual Property Organization, system of the Federal Institute of Industrial Property (Rospatent)). Literature review was performed using scientific databases: Scopus, Web of science, e-library, ScienceDirect, PubMed.

The method of microcantilever sensors was applied for the detection of C-reactive protein in buffer solution. This method is based on converting the energy of chemical reactions and molecular interactions into the elastic energy of mechanical deformation of the sensor. During the specific binding of target molecules to the receptor layer on the cantilever surface, lateral surface stress arises leading to the cantilever bending, which could be registered with high accuracy. For data analysis and interpretation the deflection of the cantilever could be recalculated into an independent characteristic - surface stress, determined by the Stoney's formula (Stoney G. G., 1909):

$$
\Delta \sigma \approx \frac{E T^{2}}{3(1-v) L^{2}} \Delta Z,
$$

where $\Delta \sigma$ is a change of surface tension, $\Delta \mathrm{Z}$ - is a relative cantilever deflection, $\mathrm{v}-$ is Poisson's ratio, E - Young's modulus of cantilever material, $\mathrm{T}$ and $\mathrm{L}$ - are the cantilever console thickness and length.

Qualitative and quantitative analysis can be performed by building calibrating plot of the relation of the surface tension and analyzed substrate concentration in the solution.

Cantilever modification with specific antibodies was performed with the utilization of superficial silane chemistry. These organosilicone compounds are capable of thin self-organizing film formation on the surface of silicon oxide. Due to a modification with functional groups (for example, aminogroups), silanes are well suited for the development of receptor layers on cantilever sensors. Experimental work was performed using silicone cantilevers Arrow TL1Au (Nanoworld, Switzerland) sized $500 \times 100 \times 1 \mu \mathrm{m}$, with resonance frequency $3-14 \mathrm{~Hz}$ (mean $6 \mathrm{~Hz}$ ) and golden reflecting coating.

Methods and development protocols for the production of receptor layer of C-reactive protein specific antibodies using aminopropyltrimethoxysilane on the surface of silicone sensors were developed.

\section{RESULTS}

Close cooperation of a team of medical and technical specialists originating from different organizations led to an elaboration of a technical solution for the development of an invasive biosensor for the prediction and early detection of diseases based on human saliva composition using implantable biosensor devices.

Development of a setup for the detection of low concentrations of proteins in liquid mediums.

Experimental setup, which allows the detection of low concentrations of target proteins in a continuous flow of analyzed medium using micromechanical cantilever sensor was developed. It is based on Bioscan microcantilever system (Biosensor Academy, Russia). This setup could simultaneously measure deflections of two cantilevers with high accuracy. Relative deflection of sensor cantilever indicates specific binding of analyte and receptor layer on its surface and signals the presence of target protein in the sample. 
The volume of measuring cell is less than 100 microliters. Laser-optical system, consisting of 15 $\mathrm{mW}$ linear laser, a system of mirrors and CCD as a detector, was used for measuring the cantilever deflection. When the cantilever deflects from the

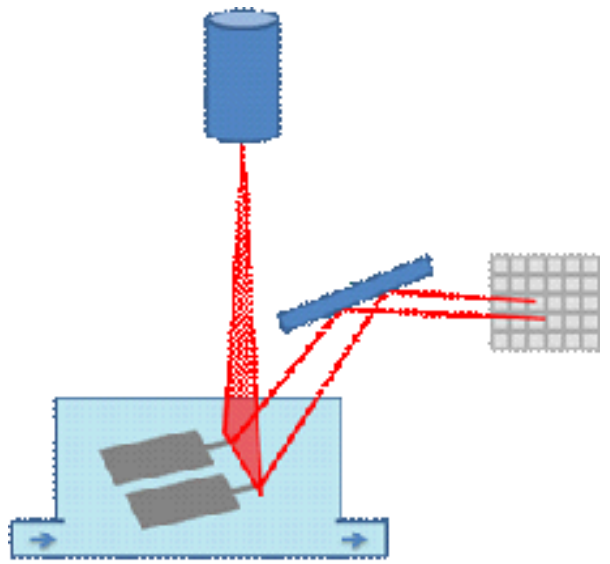

Fig. 1. Schematic layout of the operating principle of experimental setup
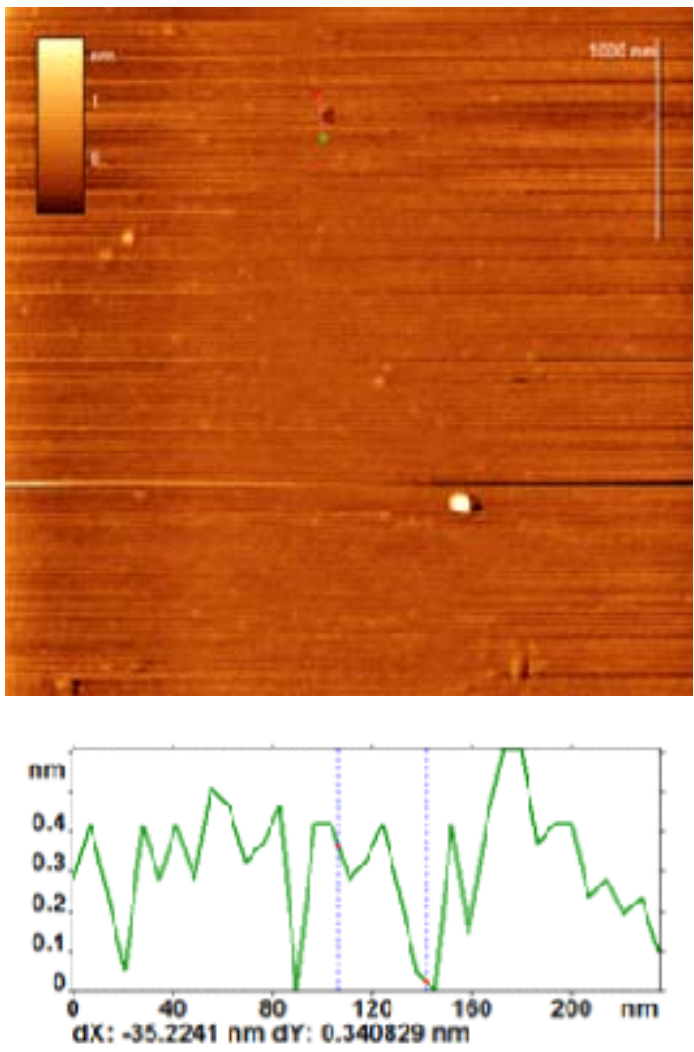

equilibrium position, corresponding beam, reflected from its free end, moves across the matrix. This movement is significantly enhanced with respect to the deflection of the cantilever due to the use of an optical arm.

The operating principle of the measuring setup is schematically shown on Figure 1.

Connection of the device to the computer and laser power supply is performed via USBinterface. In prospect miniaturization of the device will permit transition to autonomic work and remote data acquisition. Sensor data acquisition is performed using special software FemtoScan VideoSense. Measurement result is the graph of the relation of both cantilevers position over time and differential signal. Specific binding of substances from the solution with the receptor layer on cantilever surface results in a graph of kinetic analyte sorption. The pump can provide a constant flow of analyzed medium through a flowcell with a wide range of speed. In experimental conditions
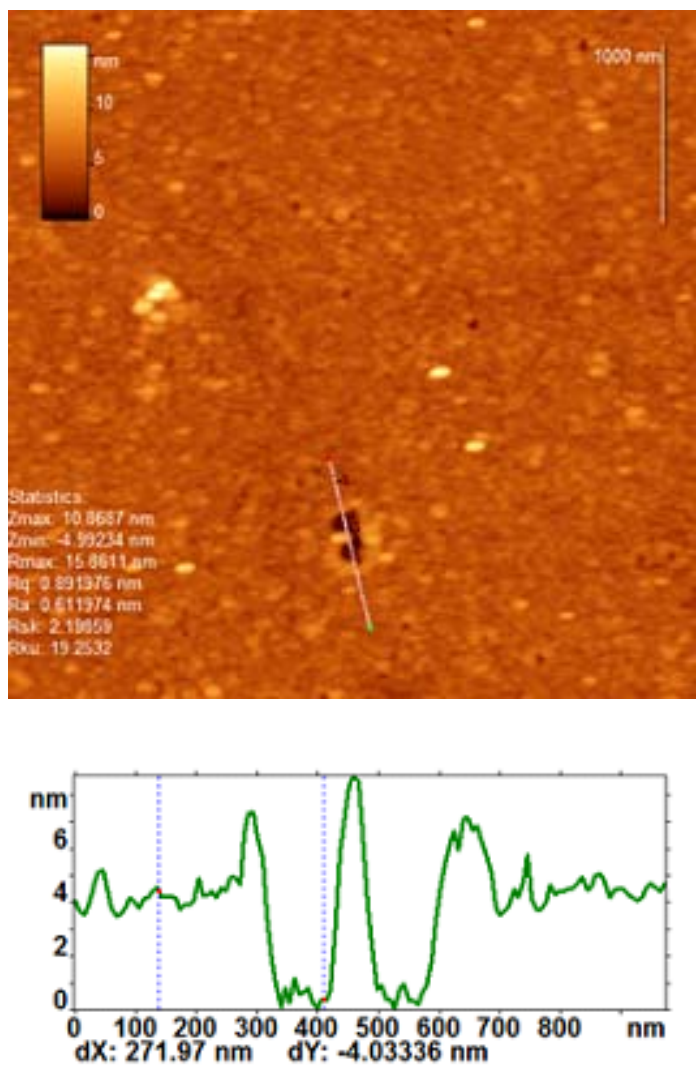

Fig. 2. AFM topology images and crossections of pure mica (left) and mica, modified with APTMS and anti-CRP antibodies (right) 
the addition of the analyte sample into the medium was performed using a two-position 6-port valve.

It is important to note, that our sensor is different from the sensor with electrical system of registration based on a piezoresistive detector presented in 2013 (Yen Y.K. et al., 2013), as it has a second abutment cantilever, which increases the accuracy of measurement in case of analyte detection with background concentration of other proteins in the sample. The range of measured concentrations remains the same, $1-200 \mu \mathrm{g} / \mathrm{ml}$ of protein.

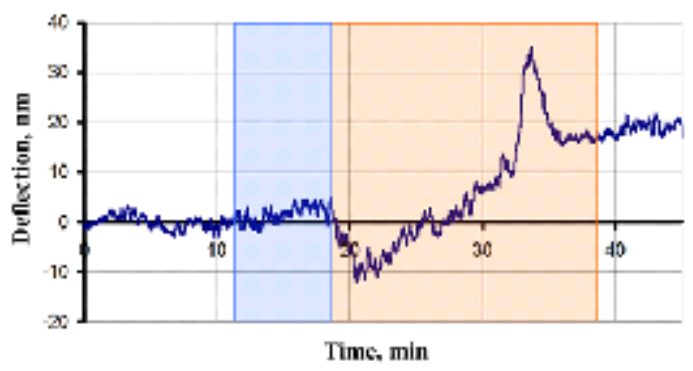

Fig. 3. The relation of relative deflection of sensor cantilever on time during CRP adsorption from the solution with concentration of $50 \mu \mathrm{g} / \mathrm{ml}$

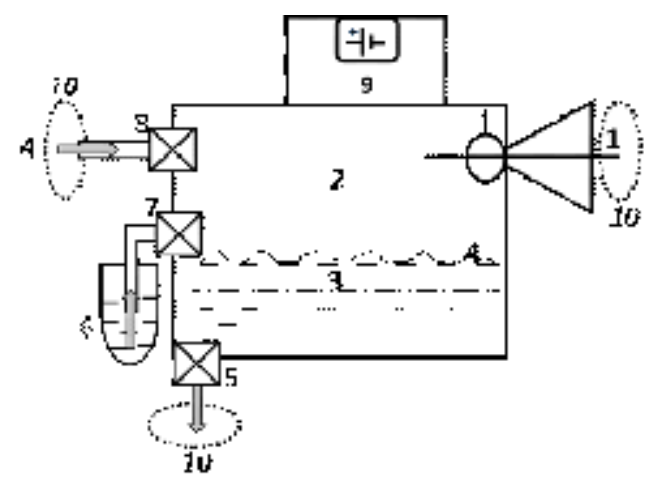

1 - micropump (micropiezofan).

2 -biochemical analysis chamber.

3 - biochemical sensor.

4 - biological fluid (saliva).

5 - microelectromechanical valve for fluid evacuation from the chamber. (efflux piezovalve).

6 - a container with rinsing fluid.

7 - microelectromechanical valve for rinsing fluid injection into the system (import piezovalve).

8 - microelectromechanical valve for biochemical fluid (saliva) injection into the chamber.

9 - microchip electronic module with battery. 10 - grid filter

Fig. 4. Microcapsule transducer

\section{Development of a method of cantilever surface modification}

Receptor layer is a key element of the sensor. It usually consists of receptor molecules and a crosslinker, which provides immobilization of receptor molecules on sensor surface. The structure of receptor layer significantly affects final signal. Firstly, crosslinker should permit unrestricted access of analyte molecules to the receptor molecules. Secondly, it should provide enough density of receptor molecules so that their interaction could lead to arising of lateral surface stress and cantilever bending. Finally, receptor layer should be protected from non-specific binding. For CRP detection with cantilever sensors we developed a receptor layer, based on monoclonal anti-CRP antibodies.

We used silicon cantilevers Arrow TL1Au (Nanoworld, Switzerland) during the experiment. One side of this cantilever is coated with thin golden layer (as a reflective coating). Thereby substances with thiol group could be used for modification of the gold surface and silane/ silatrane-based chemistry - for silicon surface modification. Thus, cantilevers could be completely immersed in modifying solution with preserved selectivity of modification. Cantilevers were modified in cuvettes with a volume of 200 microliters.

At first, the surface of cantilevers was cleaned in "piranha" solution $\left(\left(\mathrm{H}_{2} \mathrm{SO}_{4}\right)_{\text {conc }}:\left(\mathrm{H}_{2} \mathrm{O}_{2}\right.\right.$ )$_{37 \%}-1: 1$ ) for 15 minutes followed by careful rinsing in DI water and ethanol.

Silicon surface was chosen for receptor layer formation, so aminopropyltrimetoxysilane (APTMS) was used as a crosslinker for chemical binding of anti-CRP antibodies. It forms a thin aminated layer on silicon surface. Layer parameters can be modified by changing the concentration of the modifying solution and reaction duration. We found optimal parameters which permit the formation of homogeneous layer on the surface of the sensor. For that purpose the cantilevers were incubated for $1 \mathrm{~h}$ in a 10\% APTMS solution in ethanol. Then, as a result of the reaction of carboxyl-groups with amino-groups on the surface, antibodies were bound to crosslinker layer. Glutardialdehyde (GA) may be used to increase the efficacy of reaction. The sensor cantilever was immersed in $100 \mathrm{ug} / \mathrm{ml}$ solution of specific 
monoclonal antibodies in phosphate buffer solution (PBS) for 3 hours at room temperature. Reference cantilever was immersed in solution of anti-D-dimer antibodies in the same conditions. On a final stage APTMS surface which did not undergo reaction was stabilized with BSA and casein solution (1\% and $1 \% \mathrm{w} / \mathrm{w}$ ) in phosphate buffer solution for 1 hour. At the same time the opposite gold surface of cantilever was passivated due to physical adsorption. Each stage was terminated by washing with appropriate solution.

Surface modification control was performed using atomic-force microscopy method (FemtoScan, LLC RPE Advanced Technologies Center, Russia). For this purpose all stages of modification were carried out in parallel on mica platelets - a chemical analogue of silicon. Mica has atomically smooth surface which facilitates visualization and can easily be cleaved to obtain a perfectly clean surface.
Figure 2 demonstrates AFM images and crossections of pure mica surface and mica, modified with anti-CRP antibodies. From these data it is evident that surface of pure mica has roughness less than $1 \mathrm{~nm}$. The surface of receptor layer, consisting of APTMS and antibodies, has globular structure. The thickness of APTMS and antibody layer is about $4 \mathrm{~nm}$ according to atomic force microscopy.

\section{The results of surface tension measurement}

Modified cantilevers were installed into measuring cell of experimental setup, and then the cell was filled with buffer solution. Cantilevers stayed in the buffer solution for at least 8 hours to achieve equilibrium. Thereafter, a continuous flow of PBS at the rate of $1 \mathrm{ml} / \mathrm{h}$ was applied through the cell. After the difference signal reached a constant value, the valve was switched and the sample containing C-reactive protein was added into the flow. The deflections of cantilevers were

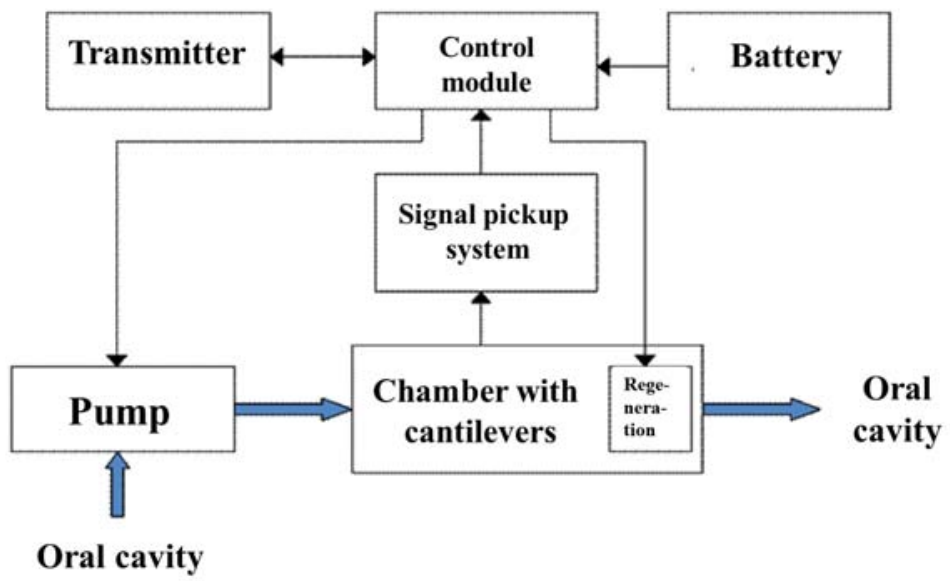

Fig. 5. Principal layout of microfluidic sensor device operation for remote monitoring of cardiovascular system parameters

measured during experiment. The relation of the relative deflection of the sensor cantilever on time, caused by specific binding of the protein from the solution with a concentration of $50 \mu \mathrm{g} / \mathrm{ml}$ is shown in Figure 3.

The first stage (blue area) corresponds to the time that takes sample to reach the measuring cell. Then (orange area) small gap, related to changes in the parameters of the medium, and a gradual increase in surface stress in the layer reaching a plateau were observed. The resulting relative deflection of cantilever sensor for the concentration of $50 \mu \mathrm{g} / \mathrm{ml}$ was about $20 \mathrm{~nm}$. This concentration approximately corresponds to the level of C-reactive protein in the blood of patients during the first hours after myocardial infarction.

\section{Micropump development}

A technical solution for the development of microcapsule transducer with a chamber for biochemical sensor and micropump controller module was established. Micropiezofan Murata MZB100 (Japan) was chosen as a basis for the development of a micropump for a sensor device due to its small size (20õ20õ2 mm) and low energy consumption (200 mWatt). Apart from that the technical solution is original. 
Design of the microcapsule transducer developed is virtually a microaspiratior with a feature of working chamber rinse. It utilizes a micropump based on a pneumatic micropiezofan, functioning as a vacuum pump in case of biological fluid injection or fluid for working chamber rinse or as a fan compressor in case of fluid evacuation from the chamber. On Figure 4 is presented a principal scheme of a technical device implanted into a dental prosthesis for a continuous monitoring of cardiovascular system based on critical changes of human saliva composition.

Operation algorithm of microcapsule device was developed, consisting of the following main stages: assay stage and preparation stage. These phases are continuously alternating, providing a constant flow through the chamber with biosensor. Operational scheme of the microcapsule transducer is as follows. Micropiezofan provides either a vacuum or a pressure in the chamber depending upon the stage of analysis. During the analysis reduced pressure is created in the chamber, which provides biological fluid (for example, saliva from the oral cavity) aspiration, which contacts the biosensor. Injection time is determined by the time of pump operation and depends on the volume needed and the speed of injection. This time is approximately two seconds (with total chamber volume $200 \mathrm{~mm}^{3}$ and effective volume of biological fluid less than $100 \mathrm{~mm}^{3}$ ). After the assay and data conversion the chamber should be rinsed and prepared for the next assay cycle. Rinsing fluid is pumped into the chamber for that purpose. Electromechanical valves made using semiconductor technology with size d"4 mm (1 for saliva evacuation, 2 - for rinsing fluid injection, 3 - for saliva aspiration into the chamber from the oral cavity) and a container with rinsing fluid are used for periodical rinsing of biochemical sensor and biological fluid evacuation from the chamber. Control of microcapsule parts is realized with a microchip electronic module, consisting of a microchip, battery, voltage converter for power supply, micropump control driver, induction charging device. Micropump power consumption is low $(0,2 \mathrm{Watt})$. Device control is performed with a sine-wave voltage with $25 \mathrm{kHz}$ frequency and amplitude 10-20 V using a special driver. This enables easy air-flow rate control and advantage of free and protracted fluid flow.
Developed prototype of a device for electromechanical biological fluid transfer for the detection of protein markers passed technical tests in buffer conditions. Positive results obtained enable to make the next step with experimental research in complex with biochemical sensor.

\section{Development of a compact sensor device}

The ultimate goal of the project is the development of an implanted biosensor device for remote monitoring of cardiovascular system parameters based on the analysis of human saliva composition. Based on the application area the main system parameters are compact size and autonomic functioning. Microfluidic format is the most promising for the development of implantable devices [X.-J. J. Li, Y. Zhou, 2013].

Microfluidic devices provide precision control of microscopic fluid volumes and can contain sensory elements. Several authors demonstrated the development of cantilever sensors as microfluidic chips [Haefliger D. et al., 2006; Johansson A.et al., 2013].

A concept of a microfluidic cantilever sensor for continuous monitoring of biological fluids was developed. The device has the following operating principle: the pump provides the flow of analyzed fluid through the chamber containing sensory and abutment cantilevers. Cantilevers have piezoresistive (or piezoelectrical) sensors. Signal pickup system consisting of amplifiers and analog-digital converters detects the position of cantilevers. Microprocessor controls the pump and signal pickup system (Figure 5).

When a positive signal of the presence of target biomarkers in the analyzed fluid is received, the microprocessor sends a signal through a transmitter to a receiver. Simultaneously a voltage applies to the regenerating electrode to regenerate the sensor. Battery is used for system power supply. We developed a concept of microfluidic cantilever chip construct, made of polydimethylsiloxane on a silicone carrier, arranged on a printed board.

\section{CONCLUSION}

In summary, our research and development project resulted in the development of a device for the analysis of biological fluids and detection of protein markers of cardiovascular 
diseases. System testing with C-reactive protein was performed. For that purpose a receptor layer based on monoclonal antibodies was developed. The system was able to detect target protein in a buffer solution with concentration $50 \mu \mathrm{g} / \mathrm{ml}$. A concept of compact sensor device based on a microfluidic chip was also developed.

Future research plan involves the broadening of analyzed marker proteins spectrum, increasing the sensitivity and transition from laseroptic to alternative deflection registration systems in order to miniaturize the device. A remote monitoring system needs to be developed, which in case of critical biomarker concentration changes will send information to an emergency medical service to substantially decrease mortality. For this purpose we need to develop a range of interfaces for digital data transfer to a computer of a medical preventive institution and/or attending physician. Such network communication protocols as WiFi and/or GPRS can be used. Besides, long-term continuous remote monitoring of cardiovascular system parameters permits the patient to control the course of therapeutic interventions and the physician to determine the efficacy of prescribed treatment and to perform timely pharmacologic correction.

It is assumed that the system will increase the quality and decrease time needed for the diagnostics of different socially significant diseases. That is why current project is relevant and up-to-date, is at the cutting edge of international research and possesses scientific novelty, dramatic significance for medical practice and economy.

\section{ACKNOWLEDGEMENTS}

Authors express gratitude for Russian Foundation for Fundamental Research (RFFR), for financial support of the research (project ${ }^{1} 13-04$ 12092).

\section{REFERENCES.}

1. Agafonova L.E., Lisitsa A.V., Shumyantseva V.V., Archakov A.I., Medical Academic Journal, 2012; 12(4): 22-24.

2. Arntz Y., Seelig J. D., Lang H.P., Zhang J., Hunziker P., Ramseyer J.P., Meyer E., Hegner M., Gerber C. Label-free protein assay based on ananomechanical cantilever array
Nanotechnology, 2003; 14: 86-90

3. Ashraf, M. W.; Tayyaba, S.; Afzulpurkar, N. Micro Electromechanical Systems (MEMS)

Based Microfluidic Devices for Biomedical Applications. Int. J. Mol. Sci. 2011; 12: 36483704

4. Boisen A., Thundat T. Design and fabrication of cantilever array biosensors, Materials today, 2009; 12(9): 32-38

5. Butt H.-J. A Sensitive Method to Measure Changes in the Surface Stress of Solids, J. Colloid Interface Sci., 1996, 180: 251-260.

6. Chammem H., Hafaid I., Meilhac O., Menaa F., Mora L., Abdelghani A. // Journal of Biomaterials and Nanobiotechnology, 2014, 5, 153-158.

7. Chen C.H.,. Hwang R.Z et. Al. A Wireless BioMEMS Sensor for C-Reactive Protein Detection Based on Nanomechanics, IEEE Trans. Biomed. Eng., 2009; 56(2): 462 -470.

8. Fritz J. Cantilever Biosensors, Analyst, 2008; 8133(7): 855-63.

9. $\quad$ Fritz J., Baller M.K., Lang H.P., Rothuizen H., Vettiger P., Meyer E., G“"untherodt H.-J., Gerber C., Gimzewski J.K. Translating Biomolecular Recognition into Nanomechanics, Science, 2000; 288: 316-318.

10. Gimzewski, J.K., Gerber, C., Meyer, E., Schlittler, R.R. Observation of a chemical reaction using a micromechanical sensor, Chem. Phys. Lett., 1994; 217: 589- 594.

11. Haefliger D., Hansen O., Boisen A. Selfpositioning of polymer membranes driven by thermodynamically induced plastic deformation, Advanced Materials 2006; 18: 238-241

12. Hoshino M., Haraguchi Y., Mizushima I., Sakai M. Recent progress in mechanical artificial pancreas. J. Artif. Organs, 2009; 12(3): 141155.

13. http://www.murata.com/en-eu/products/ mechatronics (Data 20.11.2014)

14. Ivanov M.A., Mikhaylov D.M., Starikovskiy A.V., Stupin V.A., Silina E.V. Prediction and early detection of diseases based on analysis of human saliva composition using implantable biosensor systems. Biology and Medicine, 2014; 6(3): Article ID: BM-044-14, 4 pages.

15. Johansson A., Blagoi G., Boisen A. Polymeric cantilever-based biosensor with integrated readout, Applied Physics Letters 2006; 89(17): 173505-173505-3.

16. Li P.-Y., Shih J., Lo R., Saati S., Agrawal R., Humayun M.S., Tai Y.-C., Meng E. An electrochemical intraocular drug delivery device. Sens. Actuators A Phys., 2008; 143(1): 41-48.

17. Li X.-J. J., Zhou Y. Microfluidic Devices for Biomedical Applications, Woodhead Publishing, 
2013; 676.

18. Lo R., Li P.-Y., Saati S., Agrawal R.N., Humayun M.S., Meng E. A passive MEMS drug delivery pump for treatment of ocular diseases. Biomed. Microdevices, 2009; 11(5): 959-970.

19. Nisar, A.; Afzulpurkar, N.; Mahaisavariya, B.; Tuantranont, A. MEMS-based micropumps in drug delivery and biomedical applications. Sens. Actuators B Chem., 2008; 130(2): 917-942.

20. Sabashnikov A, Popov AF, Bowles CT, Weymann A, Mohite PN, Wahlers T, Wittwer T, Zych B, Garcia-Saez D, Patil NP, Fatullayev J, Amrani M, Banner NR, Seidler T, Unsoeld B, Bireta C, Schoendube FA, Simon AR. First Experience With the Synergy Micro-Pump in Patients in INTERMACS Class $1-2$ as a Bridge to Transplantation: Pushing the Limits? Artif Organs. 2014 May 20. doi: 10.1111/aor.12316

21. Shashkanova O.Y., Melikhova E.V., Ermolayeva T.N., Sorbtsionniye i chromatograficheskiye processy. 2013; 13(5): 720-727.

22. Silina E.V., Stupin V.A. Detection of saliva composition changes in cardiovascular pathology. Terapevt, 2014; 11: 45-49.

23. Stoney, G. G. The Tension of Metallic Films Deposited by Electrolysis, Proc. R. Soc. (London), 1909; 82: 172-175.

24. Wu G., Datar R.H., Hansen K.M., Thundat T., Cote R.J., Majumdar A. Bioassay of prostatespecific antigen (PSA) using microcantilevers Nat. Biotechnol., 2001; 19: 856-860.

25. Yen Y.K., Lai Y.C., Hong W.T., Pheanpanitporn Y., Chen, C.S., Huang L.S. Electrical Detection of C-Reactive Protein Using a Single FreeStanding, Thermally Controlled Piezoresistive Microcantilever for Highly Reproducible and Accurate Measurements, Sensors 2013; 13: 9653-9668.

26. Zhang J., Lang H.P, Huber F., Bietsch A., Grange W., Certa U., McKendry R., Guntherodt H.-J., Hegner M., Gerber C. Rapid and label-free nanomechanical detection of biomarker transcripts in human RNA., Nat. Nanotechnol., 2006; 1: 214-220. 